\title{
Minimax strategies and duality with applications in financial mathematics
}

\author{
Alejandro Balbás · Raquel Balbás
}

\begin{abstract}
Many topics in Actuarial and Financial Mathematics lead to Minimax or Maximin problems (risk measures optimization, ambiguous setting, robust solutions, Bayesian credibility theory, interest rate risk, etc.). However, minimax problems are usually difficult to address, since they may involve complex vector spaces or constraints. This paper presents an unified approach so as to deal with minimax convex problems. In particular, we will yield a dual problem providing necessary and sufficient optimality conditions that easily apply in practice. Both, duals and optimality conditions are significantly simplified by drawing on the representation of probability measures on convex sets by points, classic problem for Choquet integrals. Important applications in risk analysis are given.
\end{abstract}

Keywords Optimization in Banach spaces · Min-max strategies · Duality · Applications in actuarial and financial mathematics

Mathematics Subject Classification (2000) $\quad 91 \mathrm{G} 80 \cdot 90 \mathrm{C} 47$

\section{Introduction}

The notion of general risk measure is becoming more and more important in Actuarial and Financial Mathematics. Many interesting actuarial and financial problems involve the optimization of risk measures. In a recent paper Balbás et al. [3] have provided a new method so as to optimize convex measures of risk. Useful applications of this new methodology have

\footnotetext{
A. Balbás

Académico Correspondiente de la Real Academia de Ciencias, University Carlos III of Madrid, C/ Madrid, 126, 28903 Getafe, Madrid, Spain

e-mail: alejandro.balbas@uc3m.es

R. Balbás $(\varangle)$

Department of Actuarial and Financial Economics, Complutense University of Madrid, Somosaguas Campus, 28223 Pozuelo de Alarcón, Madrid, Spain

e-mail: raquel.balbas@ccee.ucm.es
} 
been provided by the same authors. For instance, Balbás et al. [6] dealt with the optimal reinsurance problem of Actuarial Mathematics, Balbás et al. [4] dealt with portfolio choice problems and equilibrium issues in Financial Economics, and Balbás et al. [5] dealt with pricing issues in incomplete markets.

Though former papers dealt with convex analysis and sub-gradient linked optimality conditions in order to minimize risk measures (see, for instance [22]), the novelty in the analysis of Balbás et al. [3] was the incorporation of two elements: Firstly, the representation theorem of convex risk measures permits us to transform a risk minimization problem in a minimax problem. Secondly, the representation of probability measures on convex sets by points allows us to simplify the dual problem of the minimax one, as well as to provide simple (linear) necessary and sufficient optimality conditions.

There are many classical actuarial and financial problems that are beyond the minimization of risk measures but still lead to minimax or maximin problems. For instance, the minimization of distances, semi-norms or deviations, the Bayesian credibility theory as a experience rating technique [14], the incorporation of ambiguity or uncertainty ([13,24], etc.), the interest in robust solutions $([10,25]$, etc.), some interest rate linked problems $([7,9]$, etc.), etc. Therefore, it is worthwhile to study whether the Balbás et al. [3] methodology applies for further analyses.

This paper focuses on a general minimax convex problem and provides both a dual approach and necessary and sufficient optimality conditions, which easily apply in practical applications. In Sect. 2 we will introduce the general framework. Section 3 will yield a new dual problem and Theorem 3 , which states the existence of strong duality between the initial minimax problem and its dual. Some corollaries will focus on particular interesting situations. Section 4 will present four actuarial or financial applications: The optimization of risks, problems involving ambiguity and robust optimization, a portfolio choice problem involving markets with frictions, and interest rate problems. These examples are presented in order to illustrate potential applications of the developed duality theory, but we will not analyze these applications in detail because these is beyond our scope. Nevertheless, in order to improve the illustration, the dual problem and the optimality conditions of the portfolio choice problem will be obtained, and some properties of the optimal solution will be derived. Section 5 will conclude the paper.

\section{Preliminaries and notations}

Let $Y$ and $\mathscr{Y}$ be Banach spaces and $Z$ and $\mathscr{Z}$ their dual spaces. Denote by $Y \times Z \ni(y, z) \rightarrow$ $\langle y, z\rangle \in \mathbb{R}$ and $\mathscr{Y} \times \mathscr{Z} \ni(\gamma, \lambda) \rightarrow\langle\gamma, \lambda\rangle \in \mathbb{R}$ the usual bilinear maps. Suppose that $\mathscr{Y}$ is ordered by the (non necessarily pointed) convex cone $\mathscr{Y}_{+}$(and therefore $\mathscr{Z}$ is ordered by the dual cone $\mathscr{Z}_{+}$). For $j=1,2, \ldots, k$ fix $y_{j} \in Y, T_{j}: Y \rightarrow Y$ linear and continuous, and $\Delta_{j} \subset Z$ convex and $\sigma(Z, Y)$-compact. Fix finally a convex set $Y_{0} \subset Y$ and a convex function $H: Y_{0} \rightarrow \mathscr{Y}$. We will deal with the minimax problem

$$
\left\{\begin{array}{l}
\operatorname{Min} \rho(y) \\
H(y) \leq 0, \quad y \in Y_{0}
\end{array}\right.
$$

$\rho: Y \rightarrow \mathbb{R}$ given by

$$
\rho(y)=\operatorname{Max}\left\{\left\langle T_{j}(y)+y_{j}, z_{j}\right\rangle ; z_{j} \in \Delta_{j}, j=1,2, \ldots, k\right\} .
$$


Notice that the $w e a k^{*}$-compactness of every $\Delta_{j}$ guarantees the existence of the maximum in (2). In order to prevent the existence of "duality gaps" ([15], Chapter VIII), we will impose the Slater Qualification below.

Assumption $1 \mathscr{Y}_{+}$has non void interior and there exists $y \in Y_{0}$ with $H(y) \in-\left(\mathscr{Y}_{+}\right)^{\circ}$. In particular, (1) is feasible.

Expression (2) implies that

$$
\rho\left(\lambda y+(1-\lambda) y^{\prime}\right) \leq \lambda \rho(y)+(1-\lambda) \rho\left(y^{\prime}\right)
$$

for every $\lambda \in[0,1]$ and every $y, y^{\prime} \in Y$, and consequently $\rho$ is a convex function and (1) is a convex problem.

As will be seen in sect. 4, some actuarial and financial problems have the maximin form

$$
\left\{\begin{array}{l}
\operatorname{Max} \phi(y) \\
H(y) \leq 0, \quad y \in Y_{0}
\end{array}\right.
$$

where

$$
\phi(y)=\operatorname{Min}\left\{\left\langle T_{j}(y)+y_{j}, z_{j}\right\rangle ; z_{j} \in \Delta_{j}, j=1,2, \ldots, k\right\} .
$$

Obviously, (3) and (4) may be trivially reduced to (1) and (2) because one can deal with the equivalent problem

$$
\left\{\begin{array}{l}
\operatorname{Min}-\phi(y) \\
H(y) \leq 0, \quad y \in Y_{0}
\end{array}\right.
$$

and

$$
-\phi(y)=\operatorname{Max}\left\{\left\langle T_{j}(y)+y_{j}, z_{j}\right\rangle ; z_{j} \in-\Delta_{j}, j=1,2, \ldots, k\right\} .
$$

Denote by $\mathscr{C}\left(\Delta_{j}\right)$ the Banach space composed of the $\mathbb{R}$-valued $\sigma(Z, Y)$-continuous functions on $\Delta_{j}$ endowed with the usual supremum norm. Denote by $\mathscr{M}\left(\Delta_{j}\right)$ the space of inner regular real valued $\sigma$-additive measures on the Borel $\sigma$-algebra of $\Delta_{j}\left(\Delta_{j}\right.$ endowed with the weak $^{*}$ topology), and by $\mathscr{P}\left(\Delta_{j}\right) \subset \mathscr{M}\left(\Delta_{j}\right)$ the set composed of those $\sigma$-additive measures that are probabilities (i.e., if $\mu \in \mathscr{M}\left(\Delta_{j}\right)$ then $\mu \in \mathscr{P}\left(\Delta_{j}\right)$ if $\mu \geq 0$ and $\mu\left(\Delta_{j}\right)=1$ ). According to the Riesz Representation Theorem $\mathscr{M}\left(\Delta_{j}\right)$ endowed with the variation norm is the dual space of $\mathscr{C}\left(\Delta_{j}\right)$. In order to simplify some notations, $\mathscr{C}_{+}\left(\Delta_{j}\right)$ and $\mathscr{M}_{+}\left(\Delta_{j}\right)$ will represent the usual non-negative cones of $\mathscr{C}\left(\Delta_{j}\right)$ and $\mathscr{M}\left(\Delta_{j}\right)$, respectively.

Next we will present a main Lemma. We will omit the proof because this is a particular case of several results regarding the representation of probability measures on convex sets by points. For instance, a more general Proposition may be found in Phelps [19], pp. 3. ${ }^{1}$

Lemma 1 For every $\mathbb{P} \in \mathscr{P}\left(\Delta_{j}\right)$ there exists a unique $z_{\mathbb{P}} \in Z$ such that

$$
\int_{\Delta_{j}}\langle y, z\rangle d \mathbb{P}(z)=\left\langle y, z_{\mathbb{P}}\right\rangle
$$

for every $y \in Y$. Furthermore, $z_{\mathbb{P}} \in \Delta_{j}$.

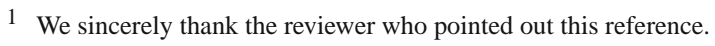


Remark 1 Let us point out that Expression (2) may be slightly relaxed. Indeed, in the line of Balbás et al. [3], we could consider the existence of $\Delta_{1}, \Delta_{2}, \ldots, \Delta_{k} \subset Z \times \mathbb{R}$, convex and compact for the product of $\sigma(Z, Y)$ and the usual topology of $\mathbb{R}$, such that the equality

$$
\rho(y)=\operatorname{Max}\left\{\left\langle T_{j}(y)+y_{j}, z_{j}\right\rangle-\alpha_{j} ; \quad\left(z_{j}, \alpha_{j}\right) \in \Delta_{j}, \quad j=1,2, \ldots, k\right\}
$$

holds for every $y \in Y$. We will not study this extension for the sake of brevity. Nevertheless, our main results may be given in the more general framework above, and the proofs are very similar to those here presented. Our proofs may be adapted to (6) by proceeding as in Balbás et al. [3].

\section{Duality for min-max problems}

Henceforth we will consider the following dual problem of (1).

$$
\left\{\begin{array}{l}
\operatorname{Max}\left(\operatorname{Inf}\left\{\left(\sum_{j=1}^{k} \lambda_{j}\left\langle T_{j}(y)+y_{j}, z_{j}\right\rangle\right)+\Lambda \circ H(y) ; y \in Y_{0}\right\}\right) \\
z_{j} \in \Delta_{j}, \quad j=1,2, \ldots, k, \quad \Lambda \in \mathscr{Z}_{+} \\
\lambda_{j} \in \mathbb{R}, \quad \lambda_{j} \geq 0, \quad j=1,2, \ldots, k, \quad \sum_{j=1}^{k} \lambda_{j}=1
\end{array}\right.
$$

In order to simplify some expressions let us denote by $D$ the dual feasible set, i.e.,

$D=\left\{\left(\left(z_{j}\right)_{j=1}^{k}, \Lambda,\left(\lambda_{j}\right)_{j=1}^{k}\right) \in \prod_{j=1}^{k}\left(\Delta_{j}\right) \times \mathscr{Z}_{+} \times \mathbb{R}^{k} ; \sum_{j=1}^{k} \lambda_{j}=1 \quad\right.$ and $\left.\quad \lambda_{j} \geq 0, \quad j=1,2, \ldots, k\right\}$

We will prove the absence of duality gap and the existence of strong duality between (1) and (7). However, the standard Duality Theory for Convex Programming generates a dual problem much more complex than (7), since some dual variables should involve spaces of inner regular $\sigma$-additive measures (recall that $\mathscr{M}\left(\Delta_{j}\right)$ is the dual of $\mathscr{C}\left(\Delta_{j}\right)$ ). Thus, let us see that Lemma 1 permits us to simplify the usual dual of (1).

\section{Lemma 2 Consider Problem}

$$
\left\{\begin{array}{l}
\operatorname{Max}\left(\operatorname{Inf}_{y \in Y_{0}}\left\{\left(\sum_{j=1}^{k} \int_{\Delta_{j}}\left\langle T_{j}(y)+y_{j}, z\right\rangle d m_{j}(z)\right)+\Lambda \circ H(y)\right\}\right) \\
\Lambda \in \mathscr{Z}_{+}, \quad m_{j} \in \mathscr{M}_{+}\left(\Delta_{j}\right), \quad j=1,2, \ldots, k \\
\sum_{j=1}^{k} \int_{\Delta_{j}} d m_{j}=1
\end{array}\right.
$$

and denote by $D_{M}$ its feasible set. Consider the correspondence

$$
\prod_{j=1}^{k}\left(\mathscr{M}_{+}\left(\Delta_{j}\right)\right) \times \mathscr{Z}_{+} \ni\left(\left(m_{j}\right)_{j=1}^{k}, \Lambda\right) \rightarrow\left(\left(z_{j}\right)_{j=1}^{k}, \Lambda,\left(\lambda_{j}\right)_{j=1}^{k}\right) \in \prod_{j=1}^{k}\left(\Delta_{j}\right) \times \mathscr{Z}_{+} \times \mathbb{R}^{k}
$$

characterized by

$$
\begin{aligned}
& \lambda_{j}=\int_{\Delta_{j}} d m_{j}, \quad j=1,2, \ldots, m \\
& \left\langle y, z_{j}\right\rangle=\frac{1}{m_{j}\left(\Delta_{j}\right)} \int_{\Delta_{j}}\langle y, z\rangle d m_{j}(z) \quad \forall y \in Y, \quad \text { if } m_{j}\left(\Delta_{j}\right)>0 \\
& z_{j} \in \Delta_{j} \text { is arbitrary, if } m_{j}\left(\Delta_{j}\right)=0
\end{aligned}
$$


This correspondence is surjective from $D_{M}$ to $D$, and the (8)-objective value in every $\left(\left(m_{j}\right)_{j=1}^{k}, \Lambda\right)$ equals the (7)-objective value in its images. Hence,

$$
\left(\left(m_{j}\right)_{j=1}^{k}, \Lambda\right) \in \prod_{j=1}^{k}\left(\mathscr{M}_{+}\left(\Delta_{j}\right)\right) \times \mathscr{Z}_{+}
$$

solves (8) if and only if

$$
\left(\left(z_{j}\right)_{j=1}^{k}, \Lambda,\left(\lambda_{j}\right)_{j=1}^{k}\right) \in \prod_{j=1}^{k}\left(\Delta_{j}\right) \times \mathscr{Z}_{+} \times \mathbb{R}^{k}
$$

solves (7), and, conversely, every solution of (7) is given by (9) and a solution of (8). Both optimal values coincide.

Proof Lemma 1 shows that the correspondence given by (9) is well defined, and the constraints of (8) show that the element $\left(\left(z_{j}\right)_{j=1}^{k}, \Lambda,\left(\lambda_{j}\right)_{j=1}^{k}\right)$ in (9) is (7)-feasible. Moreover, the objective function of $\left(\left(z_{j}\right)_{j=1}^{k}, \Lambda,\left(\lambda_{j}\right)_{j=1}^{k}\right)$ on (7) equals the objective function of $\left(\left(m_{j}\right)_{j=1}^{k}, \Lambda\right)$ on (8). Hence, the result will be proved if we see that every (7)-feasible $\left(\left(z_{j}\right)_{j=1}^{k}, \Lambda,\left(\lambda_{j}\right)_{j=1}^{k}\right)$ is generated by a (8)-feasible $\left(\left(m_{j}\right)_{j=1}^{k}, \Lambda\right)$. To prove that property, take a (7)-feasible $\left(\left(z_{j}\right)_{j=1}^{k}, \Lambda,\left(\lambda_{j}\right)_{j=1}^{k}\right)$ and define

$$
\left(m_{j}\right)_{j=1}^{k}=\left(\lambda_{j} \delta_{z_{j}}\right)_{j=1}^{k} \in \prod_{j=1}^{k}\left(\mathscr{M}_{+}\left(\Delta_{j}\right)\right),
$$

every $\delta_{z_{j}}$ denoting the usual Dirac delta concentrating the whole mass on $\left\{z_{j}\right\}$.

Theorem 3 Suppose that (1) is bounded.

a) Problem (7) is solvable (the optimal value is attainable) and both optimal objective values coincide, i.e.,

$$
\begin{aligned}
& \text { Inf } y \in Y_{0} \quad(\rho(y)) \\
& \quad H(y) \leq 0 \\
& =\operatorname{Max}_{\left(\left(z_{j}\right)_{j=1}^{k}, \Lambda,\left(\lambda_{j}\right)_{j=1}^{k}\right) \in D}\left(\operatorname{Inf}\left\{\sum_{j=1}^{k} \lambda_{j}\left(\left\langle T_{j}(y)+y_{j}, z_{j}\right\rangle\right)+\Lambda \circ H(y) ; y \in Y_{0}\right\}\right) .
\end{aligned}
$$

b) If $y^{*} \in Y_{0}$ and $H\left(y^{*}\right) \leq 0$ then $y^{*}$ solves Problem (1) if and only if there exists $\left(\left(z_{j}^{*}\right)_{j=1}^{k}, \Lambda^{*},\left(\lambda_{j}^{*}\right)_{j=1}^{k}\right) \in D$ such that

$$
\begin{aligned}
& \sum_{j=1}^{k} \lambda_{j}^{*}\left(\left\langle T_{j}(y)+y_{j}, z_{j}^{*}\right\rangle\right)+\Lambda^{*} \circ H(y) \\
& \geq \sum_{j=1}^{k} \lambda_{j}^{*}\left(\left\langle T_{j}\left(y^{*}\right)+y_{j}, z_{j}^{*}\right\rangle\right)+\Lambda^{*} \circ H\left(y^{*}\right)
\end{aligned}
$$

for every $y \in Y_{0}$, and the complementary slackness condition

$$
\left\{\begin{array}{l}
\Lambda^{*} \circ H\left(y^{*}\right)=0 \\
\lambda_{j}^{*}\left(\left\langle T_{j}\left(y^{*}\right)+y_{j}, z_{j}^{*}\right\rangle-\rho\left(y^{*}\right)\right)=0, \quad j=1,2, \ldots, k
\end{array}\right.
$$

holds. In such a case $\left(\left(z_{j}^{*}\right)_{j=1}^{k}, \Lambda^{*},\left(\lambda_{j}^{*}\right)_{j=1}^{k}\right)$ solves Problem (7). 
Proof Expression (2) shows that (1) is equivalent to

$$
\left\{\begin{array}{l}
\operatorname{Min} \theta \\
\left\langle T_{1}(y)+y_{1}, z\right\rangle-\theta \leq 0, \quad \forall z \in \Delta_{1} \\
\ldots \\
\left\langle T_{k}(y)+y_{k}, z\right\rangle-\theta \leq 0, \quad \forall z \in \Delta_{k} \\
H(y) \leq 0 \\
\theta \in \mathbb{R}, \quad y \in Y_{0}
\end{array}\right.
$$

$(\theta, y)$ being the decision variable. Indeed, $y$ solves (1) if and only if $(\theta, y)$ solves (12) for some $\theta \in \mathbb{R}$, in which case $\theta=\rho(y)$. The first constraints of (12) are $\mathscr{C}\left(\Delta_{j}\right)$-valued. Since $\mathscr{M}\left(\Delta_{j}\right)$ is the dual space of $\mathscr{C}\left(\Delta_{j}\right)$ the Lagrangian function ([15], Corollary 1, pp. 219)

$$
\mathbb{R} \times Y_{0} \times \prod_{j=1}^{k}\left(\mathscr{M}_{+}\left(\Delta_{j}\right)\right) \times \mathscr{Z}_{+} \ni\left(\theta, y,\left(m_{j}\right)_{j=1}^{k}, \Lambda\right) \rightarrow \mathscr{L}\left(\theta, y,\left(m_{j}\right)_{j=1}^{k}, \Lambda\right) \in \mathbb{R}
$$

becomes

$$
\mathscr{L}\left(\theta, y,\left(m_{j}\right)_{j=1}^{k}, \Lambda\right)=\theta\left(1-\sum_{j=1}^{k} \int_{\Delta_{j}} d m_{j}\right)+\sum_{j=1}^{k}\left(\int_{\Delta_{j}}\left\langle T_{j}(y)+y_{j}, z\right\rangle d m_{j}(z)\right)+\Lambda \circ H(y) .
$$

According to the Duality Theory in [15], Theorem 1, pp. 224,

$$
\left(\left(m_{j}\right)_{j=1}^{k}, \Lambda\right) \in \prod_{j=1}^{k}\left(\mathscr{M}_{+}\left(\Delta_{j}\right)\right) \times \mathscr{Z}_{+}
$$

is dual-feasible if and only if

$$
\operatorname{Inf}_{(\theta, y) \in \mathbb{R} \times Y_{0}} \mathscr{L}\left(\theta, y,\left(m_{j}\right)_{j=1}^{k}, \Lambda\right)>-\infty,
$$

which implies that $\sum_{j=1}^{k} \int_{\Delta_{j}} d m_{j}=1$. In such a case, $\mathscr{L}$ does not depend on $\theta$, and the dual problem of (1) becomes (8). Since the non negative cone of $\mathscr{C}\left(\Delta_{j}\right)$ has non void interior, in order to guarantee that (8) is solvable and there is no duality gap between (1) and (8) it is sufficient to see that (12) satisfies the Slater qualification, i.e., the constraints of this problem are strictly satisfied by at least a feasible element ([15], Theorem 1, pp. 224). But this is obvious because one can choose a feasible element $(\theta, y)$ with $H(y)$ in the interior of $\mathscr{Y}_{+}$ (Assumption 1) and then take

$$
\theta>\operatorname{Max}\left\{\left\langle T_{j}(y)+y_{j}, z\right\rangle ; z \in \Delta_{j}\right\},
$$

$j=1,2, \ldots, k$, so as to ensure that $\left\langle T_{j}(y)+y_{j}, z\right\rangle-\theta<0$ for every $z \in \Delta_{j}$ and $j=$ $1,2, \ldots, k$.

a) Statement $a$ ) is an obvious consequence of Lemma 2 and the existence of strong duality between (1) and (8).

b) Suppose that $y^{*} \in Y_{0}$ and $H\left(y^{*}\right) \leq 0 . y^{*}$ solves (1) if and only if there exists $\theta^{*} \in \mathbb{R}$ such that $\left(\theta^{*}, y^{*}\right)$ solves (12), in which case $\theta^{*}=\rho\left(y^{*}\right)$. According to [15], Theorem 1 , pp. 224, $\left(\theta^{*}, y^{*}\right)$ solves (12) if and only if there exists $\left(\left(m_{j}^{*}\right)_{j=1}^{k}, \Lambda^{*}\right) \in \prod_{j=1}^{k}\left(\mathscr{M}_{+}\left(\Delta_{j}\right)\right) \times$ 
$\mathscr{Z}_{+}$such that

$$
\begin{aligned}
& \sum_{j=1}^{k}\left(\int_{\Delta_{j}}\left\langle T_{j}\left(y^{*}\right)+y_{j}, z\right\rangle d m_{j}^{*}(z)\right)+\Lambda^{*} \circ H\left(y^{*}\right) \\
& \geq \sum_{j=1}^{k}\left(\int_{\Delta_{j}}\left\langle T_{j}(y)+y_{j}, z\right\rangle d m_{j}^{*}(z)\right)+\Lambda^{*} \circ H(y)
\end{aligned}
$$

for every $y \in Y_{0}$, and the complementary slackness conditions

$$
\int_{\Delta_{j}}\left(\left\langle T_{j}\left(y^{*}\right)+y_{j}, z\right\rangle-\theta^{*}\right) d m_{j}^{*}(z)=0, \quad j=1,2, \ldots, k
$$

and $\Lambda^{*} \circ H\left(y^{*}\right)=0$ hold. Besides, Lemma 2 and its proof prove that (13) holds if and only if (10) holds. Moreover, (14) and $\theta^{*}=\rho\left(y^{*}\right)$ imply the fulfillment of (11).

Conversely, if (10) and (11) hold then Lemma 2 guarantees the fulfillment of (13). If we show that (14) holds for some $\theta^{*}$ with $\left(\theta^{*}, y^{*}\right)(12)$-feasible then $y^{*}$ will solve (1). Take $\theta^{*}=\rho\left(y^{*}\right)$ and (2) guarantees that $\left(\theta^{*}, y^{*}\right)$ is (12)-feasible.

Finally, in the affirmative case, $\left(\left(m_{j}\right)_{j=1}^{k}, \Lambda\right)$ solves (8), and then

$$
\left(\left(z_{j}^{*}\right)_{j=1}^{k}, \Lambda^{*},\left(\lambda_{j}^{*}\right)_{j=1}^{k}\right)
$$

solves Problem (7) due to Lemma 2.

Consider now that Constraint $H(y) \leq 0$ is removed. The new problem becomes

$$
\left\{\begin{array}{l}
\operatorname{Min} \rho(y) \\
y \in Y_{0}
\end{array}\right.
$$

whose dual will be

$$
\left\{\begin{array}{l}
\operatorname{Max}\left(\operatorname{Inf}\left\{\left(\sum_{j=1}^{k} \lambda_{j}\left\langle T_{j}(y)+y_{j}, z_{j}\right\rangle\right) ; y \in Y_{0}\right\}\right) \\
z_{j} \in \Delta_{j}, \quad j=1,2, \ldots, k . \\
\lambda_{j} \in \mathbb{R}, \quad \lambda_{j} \geq 0, \quad j=1,2, \ldots, k, \quad \sum_{j=1}^{k} \lambda_{j}=1
\end{array}\right.
$$

We still have strong duality between (15) and (16).

Corollary 4 Suppose that (15) is feasible and bounded from below.

a) Problem (16) is solvable (the optimal value is attainable) and both optimal objective values coincide.

b) If $y^{*} \in Y_{0}$ then $y^{*}$ solves Problem (15) if and only if there exists

$$
\left(\left(z_{j}^{*}\right)_{j=1}^{k},\left(\lambda_{j}^{*}\right)_{j=1}^{k}\right)
$$

(16)-feasible such that

$$
\sum_{j=1}^{k} \lambda_{j}^{*}\left\langle T_{j}(y)+y_{j}, z_{j}^{*}\right\rangle \geq \sum_{j=1}^{k} \lambda_{j}^{*}\left\langle T_{j}\left(y^{*}\right)+y_{j}, z_{j}^{*}\right\rangle
$$

for every $y \in Y_{0}$, and the complementary slackness condition

$$
\lambda_{j}^{*}\left(\left\langle T_{j}\left(y^{*}\right)+y_{j}, z_{j}^{*}\right\rangle-\rho\left(y^{*}\right)\right)=0, \quad j=1,2, \ldots, k
$$

holds. In such a case $\left(\left(z_{j}^{*}\right)_{j=1}^{k},\left(\lambda_{j}^{*}\right)_{j=1}^{k}\right)$ solves Problem (16). 
Proof It immediately follows from the previous Theorem if one takes $\mathscr{Y}=\mathbb{R}$ and $H=-1$.

As said in the previous section, some classical problems of Actuarial and Financial Mathematics are Maximin rather than Minimax. In such a case, by using the straightforward modifications indicated in Sect. 2, we have:

Corollary 5 Suppose that (3) is bounded. Then, its dual problem is

$$
\left\{\begin{array}{l}
\operatorname{Min}\left(\operatorname{Sup}\left\{\left(\sum_{j=1}^{k} \lambda_{j}\left\langle T_{j}(y)+y_{j}, z_{j}\right\rangle\right)-\Lambda \circ H(y) ; y \in Y_{0}\right\}\right) \\
z_{j} \in \Delta_{j}, \quad j=1,2, \ldots, k, \quad \Lambda \in \mathscr{Z}_{+} \\
\lambda_{j} \in \mathbb{R}, \quad \lambda_{j} \geq 0, \quad j=1,2, \ldots, k, \quad \sum_{j=1}^{k} \lambda_{j}=1
\end{array}\right.
$$

There is no duality gap between both problems and (17) is solvable. Furthermore, if $y^{*} \in Y_{0}$ and $H\left(y^{*}\right) \leq 0$, then it solves (3) if and only if there exists

$$
\left(\left(z_{j}^{*}\right)_{j=1}^{k}, \Lambda^{*},\left(\lambda_{j}^{*}\right)_{j=1}^{k}\right)
$$

(17)-feasible such that

$$
\sum_{j=1}^{k} \lambda_{j}^{*}\left(\left\langle T_{j}(y)+y_{j}, z_{j}^{*}\right\rangle\right)-\Lambda^{*} \circ H(y) \geq \sum_{j=1}^{k} \lambda_{j}^{*}\left(\left\langle T_{j}\left(y^{*}\right)+y_{j}, z_{j}^{*}\right\rangle\right)-\Lambda^{*} \circ H\left(y^{*}\right)
$$

for every $y \in Y_{0}$ and (11) holds. In such a case $\left(\left(z_{j}^{*}\right)_{j=1}^{k}, \Lambda^{*},\left(\lambda_{j}^{*}\right)_{j=1}^{k}\right)$ solves (17).

Remark 2 Notice that $\rho\left(y^{*}\right)$ arises in (11), which might generate computational problems in some applications. However, in most of the cases the minimum value in (2) will be achieved in a unique $\left(j_{0}, z_{j_{0}}\right)$. Then

$$
\lambda_{j}^{*}\left(\left\langle T_{j}\left(y^{*}\right)+y_{j}, z_{j}^{*}\right\rangle-\rho\left(y^{*}\right)\right)=0
$$

is equivalent to

$$
\begin{aligned}
& \lambda_{j}^{*}=0, \quad j \neq j_{0} \\
& \rho\left(y^{*}\right)=\left\langle T_{j_{0}}\left(y^{*}\right)+y_{j_{0}}, z_{j_{0}}^{*}\right\rangle
\end{aligned}
$$

that may be easily applied in practice.

\section{Actuarial and financial applications}

As said in the introduction, there are many classical problems in Actuarial and Financial Mathematics fitting in the framework of this paper. Let us devote this section to presenting four examples. They will be introduced in order to illustrate potential applications of the developed duality theory, but we will not analyze these applications in detail because this is beyond our scope and would significantly enlarge the paper. Nevertheless, in order to improve the illustration, the dual problem and the optimality conditions of the third example will be obtained, and some properties of the optimal solution will be derived. 


\subsection{Risk measures, semi-norms and deviations}

General risk measures are becoming very important in finance and insurance. Artzner et al. [2] introduced the axioms and properties of the "Coherent Measures of Risk", and, since then, many authors have extended the discussion. In our setting, specially important are the expectation bounded risk measures and the deviation measures of Rockafellar et al. [20], because both are particular cases of (2) with $Y=L^{p}(\Omega, \mathscr{F}, \mathbb{P})$ and $Z=L^{q}(\Omega, \mathscr{F}, \mathbb{P}), 1 \leq$ $p<\infty, 1<q \leq \infty, 1 / p+1 / q=1$, and $(\Omega, \mathscr{F}, \mathbb{P})$ being a probability space.

The optimization of risk measures and deviations is a very complex problem that has motivated several deep analyses $([16,18,22]$, etc.), all of them related to Convex Analysis. As said in the introduction, the approach of this paper is an alternative way that significantly simplifies many applications and allows us to reach further conclusions about the analyzed problems. In Balbás et al. [4-6] one can find actuarial and financial problems involving risk measures, all of them solved with the method proposed in Balbás et al. [3], that has been extended in Theorem 3 and its corollaries.

\subsection{Ambiguity and robust optimization}

Ambiguity arises in finance and insurance if we are not sure about the real probability space reflecting the random or stochastic behavior of the variables we are interested in. Recent significant examples are Calafiore [10], Schied [24] and Zhu and Fukushima [25], where portfolio selection problems are studied.

All of these analyses fit in our framework, since, instead of dealing with "variable probability spaces" one can often fix the "true" probability space $(\Omega, \mathscr{F}, \mathbb{P})$ and then introduce the ambiguity by modifying the distribution of some random variables indicating our risk/uncertainty. For instance, let us adapt the optimal reinsurance problem of Balbás et al. [6] to the ambiguous setting. Without ambiguity the problem is as follows: Consider that the insurance company receives the fixed amount $S_{0}$ (premium) and will have to pay the random variable

$$
C \in L_{+}^{p}(\Omega, \mathscr{F}, \mathbb{P})=\left\{y \in L^{p}(\Omega, \mathscr{F}, \mathbb{P}): \mathbb{P}(y \geq 0)=1\right\}
$$

within a given period $[0, T]$ (claims). Suppose also that a reinsurance contract is signed in such a way that the company will only pay $y \in L^{p}(\Omega, \mathscr{F}, \mathbb{P})$, whereas the reinsurer will pay $C-y$. If the reinsurer premium principle is given by the continuous linear function,

$$
\pi: L^{p}(\Omega, \mathscr{F}, \mathbb{P}) \longrightarrow \mathbb{R}
$$

and $S_{1}>0$ is the highest amount that the insurer would like to pay at $T$ for the contract, then the insurance company will choose $y$ (optimal retention) so as to solve

$$
\left\{\begin{array}{l}
\operatorname{Min} \rho\left(S_{0}-y-\pi(C-y)\right) \\
\pi(C-y) \leq S_{1} \\
0 \leq y \leq C
\end{array}\right.
$$

$\rho$ representing an expectation bounded and coherent risk measure. Problem (18) may incorporate ambiguity if we consider that the total claims $C$ are ambiguous and, therefore, it may be substituted by a convex set

$$
\left\{C+\sum_{j=1}^{k} \alpha_{j} C_{j} ; \quad \sum_{j=1}^{k} \alpha_{j}=1, \alpha_{j} \geq 0, j=1,2, \ldots, k\right\},
$$


where $\left\{C_{j} ; j=1,2, \ldots, k\right\} \subset L^{p}(\Omega, \mathscr{F}, \mathbb{P})$ is fixed. In such a case (18) would become

$$
\begin{cases}\operatorname{Min} F(y) & \\ \pi\left(C+C_{j}-y\right) \leq S_{1}, & j=1,2, \ldots, k \\ 0 \leq y \leq C+C_{j}, & j=1,2, \ldots, k\end{cases}
$$

where

$$
F(y)=\operatorname{Max}\left\{\left\langle\left(S_{0}-y-\pi\left(C+C_{j}-y\right)\right), z\right\rangle ; z \in \Delta, j=1,2, \ldots, k\right\},
$$

$\Delta \subset L^{q}(\Omega, \mathscr{F}, \mathbb{P})$ being the sub-gradient of the risk measure $\rho$ (see [20]).

\subsection{Imperfect markets}

In practice transaction costs may be significant enough so as to modify the real financial markets behavior. Though linear pricing rules are usual in perfect or friction free markets [11], convex pricing rules are usual when dealing with imperfections ([12,23], etc.). Let us present an optimal portfolio selection problem that applies in markets with frictions.

Assume that $\Pi: L^{2}(\Omega, \mathscr{F}, \mathbb{P}) \longrightarrow \mathbb{R}$ is a continuous, sub-additive and homogeneous pricing rule, i.e.,

$$
\Pi\left(y_{1}+y_{2}\right) \leq \Pi\left(y_{1}\right)+\Pi\left(y_{2}\right)
$$

holds for every $y_{1}, y_{2} \in L^{2}(\Omega, \mathscr{F}, \mathbb{P})$ and

$$
\Pi(\alpha y)=\alpha \Pi(y)
$$

holds for every $\alpha>0$ and every $y \in L^{2}(\Omega, \mathscr{F}, \mathbb{P})$. Analogously, let us assume that $\rho$ : $L^{2}(\Omega, \mathscr{F}, \mathbb{P}) \longrightarrow \mathbb{R}$ is a continuous, sub-additive and homogeneous risk measure representing legal capital requirements [2]. For every $y \in L^{2}(\Omega, \mathscr{F}, \mathbb{P})$ we have that $\Pi(y)$ is the price of $y$ and $\rho(y)$ is the risk of $y$. If we add both then $\Pi(y)+\rho(y)$ provides us with the amount of money that a manager must invest so as to buy Portfolio $y$. П (y) would be invested in the purchase of $y$, whereas $\rho(y)$ would be invested in a riskless asset. ${ }^{2}$ Denote by $R \in \mathbb{R}, R>0$, the minimum expected wealth that the manager would like to reach. Then, the portfolio selection problem becomes

$$
\left\{\begin{array}{l}
\operatorname{Min} \Pi(y)+\rho(y) \\
\mathbb{E}(y) \geq R, \quad y \in L^{2}(\Omega, \mathscr{F}, \mathbb{P})
\end{array}\right.
$$

$\mathbb{E}$ denoting the mathematical expectation of every random variable.

The continuity of $\Pi$ and $\rho$, Properties (19) and (20), and the Hahn Banach Theorem allow us to prove the existence of two convex and $\sigma\left(L^{2}, L^{2}\right)$-compact subsets $\Delta_{\Pi}, \Delta_{\rho} \subset$ $L^{2}(\Omega, \mathscr{F}, \mathbb{P})$ such that

$$
\left\{\begin{array}{l}
\Pi(y)=\operatorname{Max}\left\{\mathbb{E}(y z) ; z \in \Delta_{\Pi}\right\} \\
\rho(y)=\operatorname{Max}\left\{\mathbb{E}(y z) ; z \in \Delta_{\rho}\right\}
\end{array}\right.
$$

holds for every $y \in L^{2}(\Omega, \mathscr{F}, \mathbb{P})$. Thus, it trivially follows that

$$
\Pi(y)+\rho(y)=\operatorname{Max}\left\{\mathbb{E}\left(y\left(z_{\Pi}+z_{\rho}\right)\right) ; z_{\Pi} \in \Delta_{\Pi}, z_{\rho} \in \Delta_{\rho}\right\}
$$

2 If the trader is managing a fund and investing the money of the participants, then $\rho(y)$ may be understood as a "legal reserve of money" that this trader must add so as to protect the wealth of his clients if the market evolution is not positive. 
holds for every $y \in L^{2}(\Omega, \mathscr{F}, \mathbb{P})$, and Problem (21) is a particular case of Problem (1), since $\Delta_{\Pi}+\Delta_{\rho}$ is obviously a convex and $\sigma\left(L^{2}, L^{2}\right)$-compact subset of $L^{2}(\Omega, \mathscr{F}, \mathbb{P})$.

Problem (21) obviously satisfies Assumption 1, since the homogeneity of $\mathbb{E}$ implies that it is sufficient to see the existence of $y \in L^{2}(\Omega, \mathscr{F}, \mathbb{P})$ with $\mathbb{E}(y)>0$, which is trivial. Expression (7) implies that

$$
\left\{\begin{array}{l}
\operatorname{Max}\left(\operatorname{Inf}\left\{\mathbb{E}\left(y\left(z_{\Pi}+z_{\rho}\right)\right)+\Lambda(R-\mathbb{E}(y)) ; \quad y \in L^{2}\right\}\right) \\
z_{\Pi} \in \Delta_{\Pi}, \quad z_{\rho} \in \Delta_{\rho}, \quad \Lambda \in \mathbb{R}, \Lambda \geq 0
\end{array}\right.
$$

is the dual problem of (21). Clearly,

$$
\mathbb{E}\left(y\left(z_{\Pi}+z_{\rho}\right)\right)+\Lambda(R-\mathbb{E}(y))=\mathbb{E}\left(y\left(z_{\Pi}+z_{\rho}-\Lambda\right)\right)+R \Lambda,
$$

so the infimum in (22) is bounded if and only if $z_{\Pi}+z_{\rho}-\Lambda=0$, in which case it equals $R \Lambda$. Then, (22) becomes the linear optimization problem

$$
\left\{\begin{array}{l}
\operatorname{Max} R \Lambda \\
z_{\Pi}+z_{\rho}-\Lambda=0 \\
z_{\Pi} \in \Delta_{\Pi}, \quad z_{\rho} \in \Delta_{\rho}, \quad \Lambda \in \mathbb{R}, \Lambda \geq 0
\end{array}\right.
$$

Notice that the (23)-feasible set does not depend on $R>0$. If it is empty then Theorem 3 implies that (21) is unbounded for every $R>0$. This is quite unrealistic in practice, ${ }^{3}$ so let us assume that there are (23)-feasible elements. In such a case Theorem 3 guarantees that (23) is solvable, and it is obvious that the dual solution $\left(z_{\Pi}^{*}, z_{\rho}^{*}, \Lambda^{*}\right) \in \Delta_{\Pi} \times \Delta_{\rho} \times \mathbb{R}$ does not depend on $R>0$. One can also assume that $\Lambda^{*}>0$, since otherwise the duality gap absence (Theorem 3 ) would imply that optimal value of (21) would vanish, and traders could reach the desired expected wealth without investing anything. Once again this is not realistic in practice, and meaningless from a financial perspective. Thus, bearing in mind Remark 2, the necessary and sufficient optimality conditions of Theorem 3 for (21) and (23) become

$$
\left\{\begin{array}{l}
\mathbb{E}\left(y^{*}\right)=R \\
z_{\Pi}^{*}+z_{\rho}^{*}-\Lambda^{*}=0 \\
\mathbb{E}\left(y^{*}\left(z_{\Pi}^{*}+z_{\rho}^{*}\right)\right) \geq \mathbb{E}\left(y^{*}\left(z_{\Pi}+z_{\rho}\right)\right), \quad \forall\left(z_{\Pi}, z_{\rho}\right) \in \Delta_{\Pi} \times \Delta_{\rho}
\end{array}\right.
$$

$y^{*}$ denoting the solution of (21). Thus, since $\left(z_{\Pi}^{*}, z_{\rho}^{*}, \Lambda^{*}\right)$ does not depend on $R>0$, it is sufficient to find the optimal portfolio $y_{1}^{*}$ for $R=1$, and $R y_{1}^{*}$ will be the optimal solution for every $R>0$. Moreover, the optimal (minimum) investment leading to the expected wealth $R>0$ is the linear function $\Lambda^{*} R$ of $R$.

We can finally assume the existence of additional legal constraints such as short-selling restrictions. In such a case (21) remains the same with the new condition $y \geq 0$, and, proceeding as above, (23) is almost similar but the first constraint is the inequality $z_{\Pi}^{*}+z_{\rho}^{*}-\Lambda^{*} \geq 0$. The second equation in (24) is now $\mathbb{E}\left(y^{*}\left(z_{\Pi}^{*}+z_{\rho}^{*}-\Lambda^{*}\right)\right)=0$, and the rest of conclusions remains the same.

\subsection{Interest rate risk}

Interest rate risk hedging is a classical issue in Financial Mathematics. If the Term Structure of Interest Rates (TSIR) grows (falls) then bond prices fall (grow), which implies that traders may lose money due to the TSIR evolution. Usually, duration and convexity ([17], among others) are the portfolio parameters that investors control in order to protect their wealth, but

3 If (21) were unbounded for every $R>0$ then traders could reach the desired expected wealth by investing the desired negative amount of money (i.e., by borrowing as much money as desired). Unfortunately, this is far of being realistic, and it is not so easy to become so rich. 
Bierwag and Khang [9] showed that hedged portfolios are also maximin strategies. Since then, many researchers have extended the discussion, and a very complete analysis may be found, for instance, in Barber and Copper [8] or Balbás and Romera [7]. In particular, this recent paper has developed a semi-infinite simplex like algorithm ([1]) that leads to the maximin strategy for a wide family of TSIR shifts.

Following Balbás and Romera [7], consider $n$ arbitrary bonds $B_{j}, j=1,2, \ldots, n$, and denote by $p=\left(p_{1}, p_{2}, \ldots, p_{n}\right), p_{j}>0, j=1,2, \ldots, n$, the vector of prices. Suppose that $T$ is a future date such that the bond maturities lie within the interval $[0, T]$. Suppose that $m \in[0, T]$ represents the horizon planning period and $K$ is a set of real valued functions on $[0, T]$ whose elements are admissible shocks on the TSIR. The portfolio composed of $q_{j}$ units of $B_{j}, j=1,2, \ldots, n$, will be represented by $q=\left(q_{1}, q_{2}, \ldots, q_{n}\right) \in \mathbb{R}^{n}$ and $\sum_{j=1}^{n} p_{j} q_{j}$ will be its price. If $V_{j}(k)$ is the value of $B_{j}, j=1,2, \ldots, n$, at $m$ if $k \in K$ takes place, then the real valued function $V: \mathbb{R}^{n} \times K \longrightarrow \mathbb{R}$ given by

$$
V(q, k)=\sum_{j=1}^{n} q_{j} V_{j}(k)
$$

will provide the value of $q=\left(q_{1}, \ldots, q_{n}\right)$ at $m$ if $k$ takes place.

Expression (25) implies that $V(-, k)$ is linear in the $q$ variable. We will assume that $K$ is endowed with an appropriate topology and becomes Hausdorff and compact. For instance, in [7] there are three examples, whose compactness is implied by the Ascoli-Arzelà or the Alaoglu's Theorem [21]. These examples are:

a) $K_{1} \subset C[0, T]$ is composed of those continuously differentiable functions $k$ such that $|k(t)| \leq \lambda_{1}$ and $\left|k^{\prime}(t)\right| \leq \lambda_{2}$ for every $t \in[0, T] . K_{1}$ is endowed with the compact-open topology.

b) $K_{2} \subset L^{2}[0, T]$ is composed of those functions $k$ such that $|k(t)| \leq \lambda_{1}$ and $\mid k\left(t_{2}\right)-$ $k\left(t_{1}\right) \mid \leq \lambda_{2}$ almost everywhere. $K_{2}$ is endowed with the weak topology.

c) If $K_{3}$ is a closed ball of $L^{2}[0, T]$, endowed with the weak topology.

Moreover, $V_{j}: K \longrightarrow \mathbb{R}, j=1,2, \ldots, n$, is assumed to be continuous and, therefore, it follows from (25) that $V(q,-): K \longrightarrow \mathbb{R}$ is also continuous in the $k$ variable.

Given $q \in \mathbb{R}^{n}$, we define its guaranteed value at $m$ by

$$
\bar{V}(q)=\operatorname{Min}\{V(q, k) ; k \in K\},
$$

which implies that $\bar{V}$ is the minimum of a family of linear functions. Moreover, the maximin hedging problem is given by

$$
\left\{\begin{array}{l}
\operatorname{Max} \bar{V}(q) \\
q \in Q
\end{array}\right.
$$

where the convex set $Q$ will be defined by real constraints in practical applications. They may be related to budget, short-selling or duration restrictions, liabilities, and other situations. Obviously, (26) is a particular case of (3) and therefore Corollary 5 applies. Hence, Problem (26) may be frequently solved by methods less complex than the semi-infinite simplex like algorithm of [7]. Since $q \in \mathbb{R}^{n}$ then for every fixed $k \in K$ the linear function $q \rightarrow V(q, k)$ belongs to $\mathbb{R}^{n}$ too, i.e., (26) fits in (3) with the finite-dimensional framework $Y=Z=\mathbb{R}^{n}$. Thus, the conditions of Corollary 5 apply in finite dimensions, which significantly simplifies the previous theoretical and computational analyses dealing with this problem ([8,7], etc.). 


\section{Conclusions}

This paper has provided a new Duality Theory for maximin and minimax convex problems. The major finding is Theorem 3, which guarantees strong duality between the minimax problem and its dual, as well as the existence of simple systems of equations characterizing both primal and dual solutions. With respect to former studies, this new approach significantly simplifies the optimality conditions, which become easy to apply in practice. Actuarial and Financial applications have been given.

Acknowledgments Research partially supported by "RD_Sistemas SA", "Comunidad Autónoma de Madrid" (Spain), Grant S2009/ESP - 1594, and "MICINN" (Spain), Grant ECO2009 - 14457 - C04. The authors sincerely thank the anonymous reviewers for their valuable comments and suggestions. The usual caveat applies.

\section{References}

1. Anderson, E.J., Nash, P.: Linear Programming in Infinite-Dimensional Spaces. Wiley, New York (1987)

2. Artzner, P., Delbaen, F., Eber, J.M., Heath, D.: Coherent measures of risk. Math. Finance 9, 203-228 (1999)

3. Balbás, A., Balbás, B., Balbás, R.: Minimizing measures of risk by saddle point conditions. J. Comput. Appl. Math. 234, 2924-2931 (2010)

4. Balbás, A., Balbás, B., Balbás, R.: CAPM and APT-like models with risk measures. J. Bank. Finance 34, 1166-1174 (2010)

5. Balbás, A., Balbás, R., Garrido, J.: Extending pricing rules with general risk functions. Eur. J. Oper. Res. 201, 23-33 (2010)

6. Balbás, A., Balbás, B., Heras, A.: Optimal reinsurance with general risk measures. Insur. Math. Econ. 44, 374-384 (2009)

7. Balbás, A, Romera, R.: Hedging interest rate risk by optimization in Banach spaces. J. Optim. Theory Appl. 132, 175-191 (2007)

8. Barber, J.R., Copper, M.L.: A minimax risk strategy for portfolio immunization. Insur. Math. Econ. 23, 173-177 (1998)

9. Bierwag, G.O., Khang, C.: An immunization strategy is a maxmin strategy. J. Finance 37, 379-389 (1979)

10. Calafiore, G.C.: Ambiguous risk measures and optimal robust portfolios. SIAM J. Optim. 18(3), 853-877 (2007)

11. Duffie, D.: Security Markets: Stochastic Models. Academic Press, New York (1988)

12. Jouini, E., Kallal, H.: Martingales and arbitrage in securities markets with transaction costs. J. Econ. Theory 66, 178-197 (1995)

13. Klibanoff, P., Marinacci, M., Mukerji, S.: A smooth model of decision making under ambiguity. Econometrica 73, 1849-1892 (2005)

14. Lemaire, J.: Bonus-Malus Systems in Automobile Insurance. Kluwer, Boston (1995)

15. Luenberger, D.G.: Optimization by Vector Spaces Methods. Wiley, New York (1969)

16. Mansini, R., Ogryczak, W., Speranza, M.G.: Conditional value at risk and related linear programming models for portfolio optimization. Ann. Oper. Res. 152, 227-256 (2007)

17. Montrucchio, L., Peccati, L.: A note on Shiu-Fisher-Weil immunization theorem. Insur. Math. Econ. 10, 125-131 (1991)

18. Nakano, Y.: Efficient hedging with coherent risk measure. J. Math. Anal. Appl. 293, 345-354 (2004)

19. Phelps, R.R.: Lectures on Choquet's theorem, 2nd edn. In: Lecture Notes in Mathematics 1757. Springer, Berlin (2001)

20. Rockafellar, R.T., Uryasev, S , Zabarankin, M.: Generalized deviations in risk analysis. Finance Stoch. 10, 51-74 (2006)

21. Rudin, W.: Functional Analysis. McGraw-Hill, New York (1972)

22. Ruszczynski, A., Shapiro, A.: Optimization of convex risk functions. Math. Oper. Res. 31(3), 433-452 (2006)

23. Schachermayer, W.: The fundamental theorem of asset pricing under proportional transaction costs in finite discrete time. Math. Finance 14(1), 19-48 (2004)

24. Schied, A.: Optimal investments for risk- and ambiguity-averse preferences: a duality approach. Finance Stoch. 11, 107-129 (2007)

25. Zhu, S., Fukushima, M.: Worst case conditional value at risk with applications to robust portfolio management. Oper. Res. 57(5), 1155-1168 (2009) 